\title{
Curation Technologies for Cultural Heritage Archives
}

\author{
Analysing and transforming a heterogeneous data set into an interactive curation workbench
}

\author{
Georg Rehm \\ DFKI GmbH \\ Berlin, Germany \\ georg.rehm@dfki.de \\ Julián Moreno-Schneider \\ DFKI GmbH \\ Berlin, Germany \\ julian.moreno_schneider@dfki.de
}

\author{
Martin Lee \\ Freie Universität Berlin \\ Berlin, Germany \\ martin.lee@fu-berlin.de \\ Peter Bourgonje \\ DFKI GmbH \\ Berlin, Germany \\ peter.bourgonje@dfki.de
}

\begin{abstract}
We present a platform that enables the semantic analysis, enrichment, visualisation and presentation of a document collection in a way that enables human users to intuitively interact and explore the collection, in short, a curation platform or workbench. The data set used is the result of a research project, carried out by scholars from South Korea, in which official German government documents on the German re-unification were collected, intellectually curated, analysed, interpreted and published in multiple volumes. The documents we worked with are mostly in German, a small subset, mostly summaries, is in Korean. This paper describes the original research project that generated the data set and focuses upon a description of the platform and Natural Language Processing (NLP) pipeline adapted and extended for this project (e.g., OCR was added). Our key objective is to develop an interactive curation workbench that enables users to interact with the data set in several different ways that go beyond the current version of the published document collection as a set of PDF documents that are available online. The paper concludes with suggestions regarding the improvement of the platform and future work.
\end{abstract}

\section{CCS CONCEPTS}

- Information systems $\rightarrow$ Entity resolution; Data mining; Digital libraries and archives; Document representation; Retrieval tasks and goals; - Applied computing $\rightarrow$ Digital libraries and archives; Document management.

ACM Reference Format:

Georg Rehm, Martin Lee, Julián Moreno-Schneider, and Peter Bourgonje. 2019. Curation Technologies for Cultural Heritage Archives: Analysing and transforming a heterogeneous data set into an interactive curation workbench. In 3rd International Conference on Digital Access to Textual Cultural Heritage (DATeCH2019), May 8-10, 2019, Brussels, Belgium. ACM, New York, NY, USA, 6 pages. https://doi.org/10.1145/3322905.3322909

Permission to make digital or hard copies of all or part of this work for personal or classroom use is granted without fee provided that copies are not made or distributed for profit or commercial advantage and that copies bear this notice and the full citation on the first page. Copyrights for components of this work owned by others than the author(s) must be honored. Abstracting with credit is permitted. To copy otherwise, or republish, to post on servers or to redistribute to lists, requires prior specific permission and/or a fee. Request permissions from permissions@acm.org.

DATeCH2019, May 8-10, 2019, Brussels, Belgium

(C) 2019 Copyright held by the owner/author(s). Publication rights licensed to ACM. ACM ISBN 978-1-4503-7194-0/19/05 ..\$15.00

https://doi.org/10.1145/3322905.3322909

\section{INTRODUCTION}

With more and more digital content being added to the enormous collection of online news, social media, archives etc. every single day, making sense of this pile of information is getting increasingly important and challenging. Not only is new content being generated continuously, existing legacy information in analogue formats is converted into the digital realm for purposes of preservation and sustainability. This conversion of, for example, physical archives, into machine-readable formats is an important first step towards making sure the information is retained for future generations. The subsequent step, semi or maybe even fully automatically making sense of large volumes of content to enable humans quickly to grasp what is contained on a rather high level, and providing entry points for a more detailed exploration, i. e., the aggregation, sorting and re-arranging of both new and digitised information is what we perceive to be at the core of digital curation technologies [4, 19, 25-27]. In this contribution we focus on importing, analysing and curating a cultural heritage archive in the form of a very large collection of PDF documents. Section 3.1 explains the background of this collection. Afterwards, a series of prototypical text analytics workflows is described (Section 4) and the challenges of applying them to a Digital Humanities and Social Sciences data set in a from an NLP perspective - resource-rich language (German) and an under-resourced language (Korean) are discussed. The results of our analyses are visualised in a dashboard (Section 5). Conclusions and pointers to future work are presented in Section 6.

\section{RELATED WORK}

Our technical focus is on putting together various semantic analysis and visualisation components. We do not aim to improve the individual components, but by putting them together in workflows and combining them with ready-to-be-used interactive visualisations we aim to offer added value. In this brief overview of related work we concentrate on platforms that also offer functionality to import and analyse complete corpora, and visualise the results.

Several such platforms exist, they often have their own specific domain or target use case. SUMMA ${ }^{1}$ focuses on broad coverage of language through the use of Machine Translation. The NewsReader project ${ }^{2}$ similarly takes large volumes of news data and analyses it

\footnotetext{
${ }^{1}$ http://www.summa-project.eu

${ }^{2}$ http://www.newsreader-project.eu
} 
for more intuitive exploration. The HumaReC project $^{3}$ comes with an OCR component and intuitive visualisation functionality, but focuses on continuously updated content and explores a test case with a Greek, Latin and Arabic version of the new testament. The DIVE+ tool [10] implements an event-extraction based approach and supports Linked Data. Within DARIAH, several relevant platforms were developed, typically each with their own specific use case (either regarding domain, language, user base, focus on visualisation, collaborative working, etc.). Some examples are TextGrid ${ }^{4}$, ConedaKOR $^{5}$ and Geo-Browser ${ }^{6}$. Furthermore, for resources and tools created in the context of CLARIN, a comprehensive overview up until 2014 is described in [16].

Several platforms are focused on the annotation of documents. WebAnno [11] is a web-based annotation tool including various layers of morphological, syntactical, and semantic annotations. It supports roles such as annotator, curator, and project manager. ANNIS [31] (Annotation of Information Structure) is a browserbased search and visualisation architecture for linguistic multi-layer corpora with diverse types of annotation. It allows annotations on many levels as syntax, semantics, morphology and more.

Other works focus on e-science and specific Digital Humanities workflows. Weblicht $[8,15]$ is an environment for automatic annotation of text corpora. Tokenizers, taggers, and parsers are encapsulated as web services, which can be combined into processing chains. It also includes visualisations for annotations. The OpenMindTed platform [23] aims to bring full-text Open Access scholarly content from a wide range of providers together with Text and Data Mining tools from various NLP frameworks in an integrated environment. Researchers can search, select publications or upload their own corpora, process content, view the results, and download and publish the processing results. The Alveo Virtual Laboratory [5] is focused on building a platform for collaborative e-science around data representing human communication and the tools that researchers use in their analysis. It features a web-based API that provides access to data and tools.

Another class of systems is focused upon back-end processing, especially the interconnection of components and the definition of workflows, such as Apache UIMA [12], that aims at analysing large volumes of unstructured information to extract knowledge. UIMA enables applications to be set up of various components (language identification, language segmentation, entity detection, etc.), defining data flows between them, see [3] for a comparison with different systems. Another example is TextFlows [24], a text mining and NLP platform supporting the visual construction, sharing and execution of workflows.

The last group of systems relates primarily to the development of new NLP applications, such as GATE or LAPPS GRID. GATE [9] is a widely used text analysis tool. It includes a desktop client for developers, a workflow-based web application, a Java library, an architecture and a process for the creation of services. Visualisations are available, but they are not interactive. The Language Applications (LAPPS) Grid [17] is an open platform for research

\footnotetext{
${ }^{3}$ https://humarec.org

${ }^{4} \mathrm{https} / / /$ textgrid.de/en/

${ }^{5}$ https://github.com/coneda/kor

${ }^{6} \mathrm{https}: / /$ geobrowser.de.dariah.eu
}

and development involving several aspects of NLP. It enables service discovery, composition, and reuse and promotes sustainability, manageability, usability, and interoperability of NLP components. LAPPS Grid provides access to basic processing tools and resources and enables pipelining, as well as composite services such as question answering and machine translation.

\section{THE CULTURAL HERITAGE ARCHIVE}

We work with a large, heterogeneous and self-contained set of documents about the German unification, collected and digitised in the project "Sharing German government's documents on unification and Integration, and Building a data-base on german unification" at the Institute of Korean Studies at Freie Universität Berlin, between 2010 and 2016. The objective of this project, funded by the Ministry of Unification of the Republic of Korea, was to collect official political documents regarding the German reunification process to make them available for research and planning processes in the context of a potential future reunification process of Korea. The collection consists of transcripts of debates in the German Parliament, minutes of committee meetings, reports, proceedings and other document types. All primary documents are written in German, while the researchers who originally worked in the above mentioned project added summaries and analyses of the primary documents in both German and Korean. The data set contains more than 100,000 pages spread over 51 volumes/editions.

\subsection{Original Research Project and Data Set}

After the Second World War both Korea and Germany were divided. While Germany achieved formal unification in 1990, Korea remains divided. There is no dependable prognosis, as to if, when and how Korea will be unified. Korean Unification without doubt is and will be a unique case in itself; a transfer of the German model and experience to Korea is neither feasible nor possible. But there are de facto a number of important issues and areas, which nonetheless bear special relevance to Korea. To identify them as well as to conduct new research in those issues and areas, the South Korean government funded the project "Sharing German government's documents on unification and Integration, and Building a data-base on german unification" from 2010-2016 with Prof. Dr. Eun-Jeung Lee, Freie Universität Berlin, as project lead. ${ }^{7}$ The German Reunification stands as an example of integration and transformation within a country, embedded in domestic and foreign policy contexts, administrative action and the objectives and lifestyles of its citizens. Thus, it is not surprising that there exists a barely manageable amount of analyses regarding the German Reunification focusing on different aspects and opinions. The main focus of the project was a more systematic overview of selective processes after the formal reunification (with an emphasis on the micro-level dealing with practical implementation) bearing special relevance to Korea.

The main objectives of the project were:

- to select archive materials, case studies, identify relevant researchers, and commission new research on the chosen case studies;

- to identify those reunification processes after the formal reunification that bear special relevance for the Korean case;

$\overline{{ }^{7} \text { https://www.geschkult.fu-berlin.de/e/tongilbu/index.html }}$ 
- to compile a selected bibliography of existing research on German reunification;

- to conduct interviews with relevant key figures of the German reunification;

- to structure and edit a series of volumes with archive material and official documents.

The collected data was curated into 51 volumes covering a wide range of issues in the unification process. ${ }^{8}$ The data set is one of the most comprehensive compilations of material on the German reunification. In Korea in particular, the results were very positively received. Special volumes with translated documents and commentaries have been published in Korea, where they are used as a basis for further research by reunification experts. Workshops were also organised for Korean officials and academics.

In Germany, there is also an increased interest in better accessibility of data material, especially from historians, political scientists and sociologists. The results of the project are currently made available online as a set of HTML and PDF documents. The options of navigating this valuable data set and accessing specific information are rather limited, which is why, as a first step, work is now underway on a generally available web interface that will offer various formal and thematic entry points. Additional, the idea was conceived to transform the original data set into an interactive workbench that enables the further, interactive curation of this vast set of research data. The goal of the project reported upon in this paper, is to apply a set of semantic technologies to this cultural heritage archive in order to analyse and transform the data set and to provide an interactive way of accessing the collection. The curation technologies are developed to offer greater insight into temporal, geographical, entity-related and thematic contexts. The research reported in this contribution is conducted jointly by the host organisation of the original project, Freie Universität Berlin, and the German Research Centre for Artificial Intelligence (DFKI). To further increase the accessibility of the original data set the Institute of Korean Studies approached its library where a new Digital Humanities project was launched in late 2018. The Campus Library has a focus on the use and interoperability of non-latin scripts [2].

\subsection{The Data Set - Statistics and State of Play}

We are working with the results of the project described in Section 3.1, which is available as a collection of PDF files, text files, spreadsheet tables and other file formats. Since the essential information was contained in the collection of PDF files, this is where we started. It comprised almost 10 Gigabytes of data, distributed over 200 files containing over 138,000 pages in both German and Korean. The majority of pages is in German ( 96,000 pages, 38 million words), a smaller set in Korean (41,000 pages, 15 million words). Most of the PDF pages are of high quality and the recognition of individual characters/words poses no concrete challenges, at least for German (handwriting or otherwise challenging scripts occur only rarely). We apply our pipeline to this set of 138,000 pages and 53 million words, as described in the following.

\footnotetext{
${ }^{8}$ Available in PDF format at https:/www.geschkult.fu-berlin.de/e/tongilbu/ publikationen/index.html; about half of these volumes have been published as Korean editions, see https://www.geschkult.fu-berlin.de/e/tongilbu/publikationen/ koreanische-edition/index.html.
}

\section{GENERIC CURATION TECHNOLOGIES}

In this contribution we focus on the set of PDF files (Sections 3.1 and 3.2). To enable humans to quickly get an overview of what is inside this subset of the document collection, we apply a processing pipeline including Optical Character Recognition (OCR), Named Entity Recognition (NER) and document clustering. The results of the processing steps are visualised in an interactive dashboard that allows the free exploration and, ultimately, curation of the collection in an intuitive way. The multiple semantic analysis steps can be conceptualised as a way of enriching the data set with additional layers of metadata about the contents, also contextualising the document collection's contents using Linked Data [14].

\subsection{Overview of the Pipeline}

The following subsections provide an overview of the individual components applied to the corpus.

\subsection{Optical Character Recognition}

Since we are working with PDF files, the first step is to convert their content to a format suitable for text processing procedures. We implemented Apache Tesseract [29] to convert the PDF content to plain text. The most recent Version 4.0 includes language models for over 130 languages and over 35 different scripts. We obtained the best results using Version 4.0 for German and 3.5 for Korean and used the respective models directly (without any additional training) $)^{9}$.

The output of the OCR procedure is what we are working with (as input) for subsequent processing steps. We performed a limited manual evaluation (creating gold standard or ground truth files) for German and Korean, dividing the data into four categories that are representative for the entire collection. In total, the German files in these categories contained 2,870 words and the Korean files contained 2,483 words. The results for Word Error Rate (WER) and Character Error Rate (CER) are shown in Table 1. The majority of the text in the total collection is in German and for Korean, two of the four categories were not present in the collection.

\begin{tabular}{|l|c|c|c|c|}
\hline \multicolumn{1}{|c|}{ Content Type } & \multicolumn{2}{c|}{ German } & \multicolumn{2}{c|}{ Korean } \\
\hline Machine-readable (one column) & WER & CER & WER & CER \\
\hline Machine-readable (two columns) & 65.73 & 2.05 & 43.19 & 19.27 \\
\hline Tables & 56.64 & 20.72 & 85,39 & 68,99 \\
\hline Typewriter scans & 12.23 & 2.24 & - & - \\
\hline
\end{tabular}

Table 1: OCR results for German and Korean

The results varied greatly depending on content type. As is reflected by the difference in WER and CER, the quality (in terms of character readability) did not present the biggest challenge. Especially for the single column formats, CER is comparably low, although considerably higher for Korean than it is for German. The main problem, though, was formatting, as is reflected by the increase in error rates (both WER and CER) for the two colum

\footnotetext{
${ }^{9}$ See https://github.com/tesseract-ocr/tesseract/wiki for more information on supported languages, scripts and other details.
} 
and table formats. The ground truth files were created using Transkribus ${ }^{10}$ and the scores were generated using the ocrevalUAtion tool $^{11}$. For practical purposes, this was done in plain text. We expect to be able to tackle the layout format partially by exporting to a more sophisticated format (like XML or hOCR), which may be able to retain structure in a more adequate and explicit way. While improving the output for challenging formats would obviously be beneficial, for our downstream applications specifically, the impact remains relatively limited. First of all, the majority of content is single column. Second, the subsequent applications in our pipeline may not be that much affected by the incorrect interpretation of individual rows in a table, but rely on the presence or absence of specific entities, for example, in an entire document. The nature of content in tables (numbers, statistics etc.) may be less relevant for our analyses, which are more biased towards full text, however, it would surely be beneficial to include a robust procedure for text structure or layout recognition to take content components such as tables or figures into account.

\subsection{Named Entity Recognition}

The next step in the pipeline is performing NER on the OCR output. Due to better support for German, we first applied the subsequent steps in the pipeline to German. In Section 4.6, we describe our first steps toward enriching the Korean texts using Machine Translation and annotation projection. For entity spotting we implemented OpenNLP [1] and use the corpus described in [20] as training data, which contains over 1.1 million articles comprising close to 400 million tokens. With no training data available for the specific domain covered in our corpus, we used this data set to train German models for the entity types person, location and organisation. The F scores for these three types for the corpus described in [20] are 97.5, 96.9 and 87.4 respectively (we have not generated gold standards for our own corpus for this sub-task).

\subsection{Entity Linking}

After identifying the entities, we proceed with retrieving a unique identifier (URI) for them. In the current prototype, this component is using the DBPedia SPARQL endpoint ${ }^{12}$. If a URI could be retrieved (the most likely reasons for not retrieving one are either because no Wikipeda or DBpedia entry exists for this particular entity, or our implementation faced a time-out of the SPARQL endpoint), it was stored as part of the entity annotation. For our prototype, we then proceed with document clustering. Having a DBPedia URI, however, allows the retrieval of related information (such as geographical co-ordinates for location-type entities, birth-dates for person-type entities, etc.), which could be exploited further. An example of this, exploiting geographical co-ordinates is described in [27]. We plan to include similar functionality in later stages of the system.

In addition, while the current platform that is used for Entity Linking is DBPedia (due to its convenient SPARQL functionality), we plan to replace this with the Gemeinsame Normdatei (GND) ${ }^{13}$, as this is designed specifically for German, can be expected to include

\footnotetext{
${ }^{10}$ https://transkribus.eu/Transkribus/

${ }^{11} \mathrm{https}: / /$ sites.google.com/site/textdigitisation/ocrevaluation

${ }^{12}$ https://dbpedia.org/sparql

${ }^{13}$ https://www.dnb.de/DE/Standardisierung/GND/gnd_node.html
}

many more German-specific entries and also different (orthographical) variations and aliases. The results of NER are visualised in the GUI with colour-coded labels for entities as shown in Figure 1 and further discussed in Section 5.

\subsection{Document Clustering}

After entities have been spotted and a URI has been successfully retrieved, we proceed with clustering the documents by their entity URIs. We implemented WEKA [13] for this purpose. The idea behind this is that documents which contain many mentions of locations in a certain region will end up in a cluster of their own, or documents containing many mentions of specific institutions end up in their own cluster; while our clustering algorithm is unaware of these entity types, and clusters may be formed according to different dimensions (i. e., spatial, temporal, or others), the clusters that arise should help the person exploring the data set to go about this in a thematic way. The clusters are also visualised in the GUI and displayed in Figure 2.

\subsection{Enriching Korean Data}

As mentioned in Section 4.2, OCR performs worse for Korean than for German. Support for other typical NLP procedures is also considerably less common. An initiative to bundle Korean resources ${ }^{14}$ refers to a part-of-speech tagger [6], parsers ([18] and [22]) and Machine Translation evaluation data [21]. Some approaches for NER exist and are described, for example in [30] and [7]. Nevertheless, any readily available, off-the-shelf tools for NER (the next step after OCR in our pipeline) for Korean do not appear to exist. We plan to turn to annotation projection as a means of getting Korean text enriched with entities. By exploiting a parallel corpus ${ }^{15}$, we aim to train an NER system on Korean, by projecting found entities in the English text to the Korean sentences. This is currently work in progress, though, which is why we do not have any concrete results to report upon yet.

\section{INTERACTIVE CURATION WORKBENCH}

In order to allow a user to intuitively explore the data set, we implemented various means of visualising the processing results. This section includes several screen shots of the first experimental prototype. After uploading a collection, the user can browse through files and folders of the collection. Currently, this relatively basic part of the platform allows folder navigation. The file explorer will be improved by presenting the typical metadata like file size and type, last modification date, user access rights, etc. Additionally, we will add metadata produced by our semantic analyses (once this has been performed), such that users, while navigating and exploring the collection, can see features like language, number of pages, words, entities recognised, etc. After the successful upload, the user can process the collection using the pipeline with the click of a button. In the current prototype, we applied a hard-coded pipeline of NER, Entity Linking and document clustering. In the future, this feature will be modified to enable users to select specific processes, and also other parameters such as domain (relevant for NER), the Entity Linking ontology that is used (e. g., GND or DBpedia) and

\footnotetext{
${ }^{14} \mathrm{https} / /$ sites.google.com/site/koreanlanguageresources/

${ }^{15}$ See https://github.com/jungyeul/korean-parallel-corpora for example.
} 


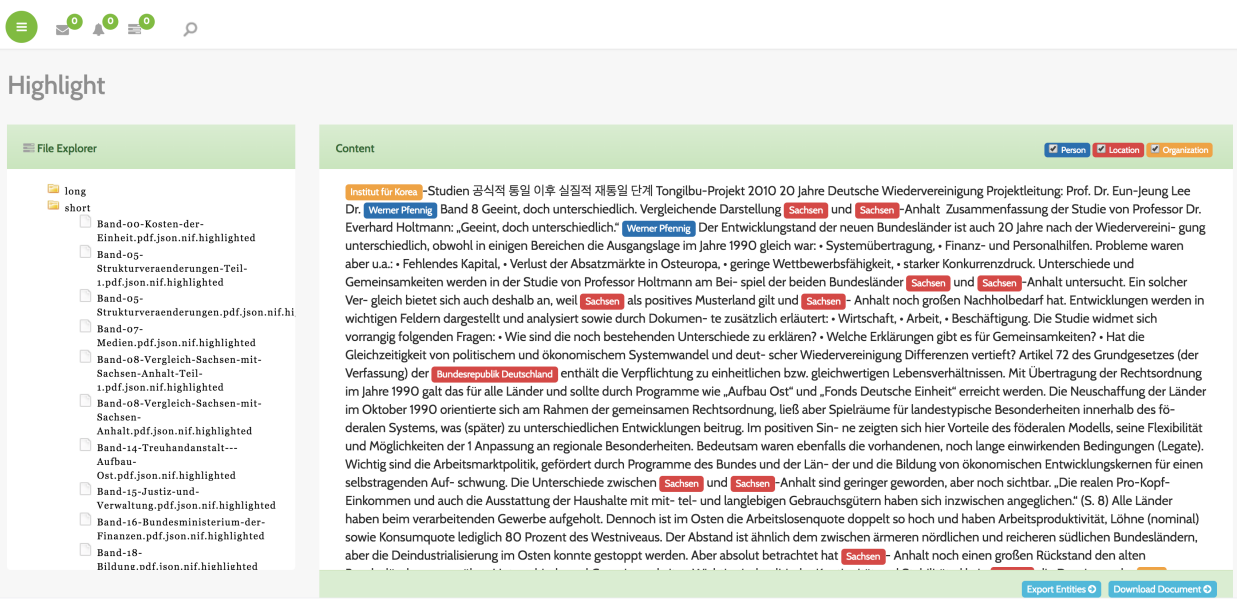

Figure 1: Visualisation of highlighted entities in a text

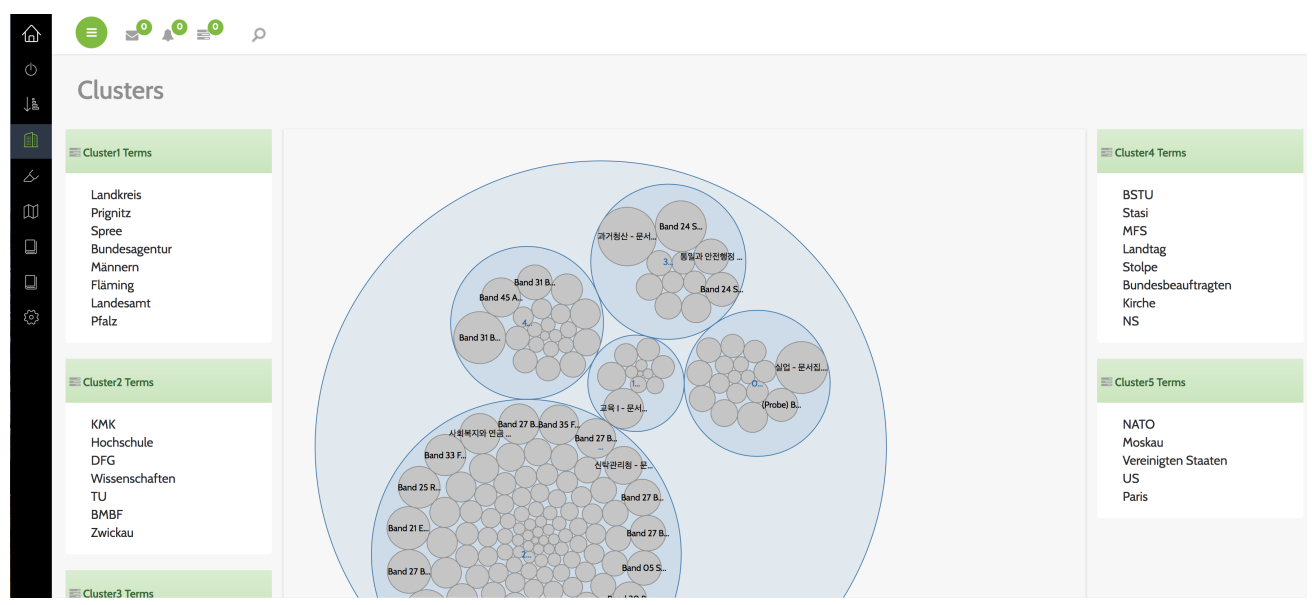

Figure 2: Visualisation of clusters of documents

other process-specific settings. We are currently implementing this Curation Workflow Manager in a project that deals with documents from the legal domain [28]. After document clustering, we visualise the data collection according to the clusters found, as in Figure 2. The panes to the left and right display the most frequent terms found in the particular cluster, the circles in the middle represent the clusters, where the user can zoom in on a cluster to see the names of the files belonging to this cluster. Zooming in on individual files, the text of a processed file with a colour-coded tagging of found entities (blue for persons, red for locations and orange for organisations) is displayed in Figure 1.

\section{SUMMARY AND CONCLUSIONS}

We describe a platform and dashboard for the curation of cultural heritage archives. It allows for the intuitive and fast analysis, exploration and visualisation of the data by exploiting recognised entities and their URIs retrieved through entity linking and operates on German (for Korean, we currently only support OCR). Our goal is to add value by combining the different semantic analyses components and making them interoperable rather than improve on individual components alone. The data we are working on comprises a collection of documents on the reunification of Germany and was the result of a project funded by the Ministry of Unification of the Republic of Korea. The collection contains German and Korean documents. The platform is work in progress and we aim to include more semantic analysis types, such as topic detection, full text search based on entities and semantic (document, and potentially paragraph) similarity. For German analyses, we consider gathering more domain-specific training data for individual components an important aspect of future work. Due to limited NLP tool support for Korean, we will experiment with Machine Translation in combination with annotation projection to bridge this gap.

The research group that is currently developing the underlying software platform in various projects is also concerned with the paradigm of Open Science and the overarching question, to what extent generic non-domain-specific tools can be productively used for carrying out or supporting concrete research processes in specific scientific areas or fields. This question applies to the prototype 
described in this paper - to what extent can off-the-shelf tools be used to implement a curation dashboard for a large and quite heterogeneous cultural heritage archive such as the result of the project "Sharing German government's documents on unification and Integration, and Building a data-base on german unification"? In other words, how far can we go with generic tools? When are domainspecific, customised tools necessary? While a general, ready-made answer cannot be given, we can make sure to make the adaptation of generic tools to specific domains as easy as possible. This aspect will be investigated more deeply in the future. In the meantime, we are convinced that the novel application, exploitation and visualisation of the processing results of existing, generic tools is feasible and helpful for users interested in further exploring a large and diverse document collection. Accordingly, we plan to carry out a user study once the prototype has been completed.

\section{REFERENCES}

[1] Apache. 2010. OpenNLP. http://opennlp.apache.org

[2] Esther Asef, Cosima Wagner, and Martin Lee. 2019. Workshop Report NonLatin Scripts in Multilingual Environments: Research Data and Digital Humanities in Area Studies. https://blogs.fu-berlin.de/bibliotheken/2019/01/18/ workshop-nls2018/.

[3] Mathias Bank and Martin Schierle. 2012. A Survey of Text Mining Architectures and the UIMA Standard.. In Proceedings of the Language Resources and Evalua tion Conference 2012 (LREC 2012), Nicoletta Calzolari, Khalid Choukri, Thierry Declerck, Mehmet Ugur Dogan, Bente Maegaard, Joseph Mariani, Jan Odijk, and Stelios Piperidis (Eds.). European Language Resources Association (ELRA), 3479-3486. http://dblp.uni-trier.de/db/conf/lrec/lrec2012.html\#BankS12

[4] Peter Bourgonje, Julian Moreno-Schneider, Jan Nehring, Georg Rehm, Felix Sasaki, and Ankit Srivastava. 2016. Towards a Platform for Curation Technologies: Enriching Text Collections with a Semantic-Web Layer. In The Semantic Web (Lecture Notes in Computer Science), Harald Sack, Giuseppe Rizzo, Nadine Steinmetz, Dunja Mladenia, Sören Auer, and Christoph Lange (Eds.). Springer, 65-68. ESWC 2016 Satellite Events. Heraklion, Crete, Greece, 2016 Revised Selected Papers.

[5] Steve Cassidy, Dominique Estival, Timothy Jones, Denis Burnham, and Jared Burghold. 2014. The Alveo Virtual Laboratory: A Web Based Repository API. In Proceedings of the Ninth International Conference on Language Resources and Evaluation, LREC 2014, Reykjavik, Iceland, May 26-31, 2014., Nicoletta Calzolari, Khalid Choukri, Thierry Declerck, Hrafn Loftsson, Bente Maegaard, Joseph Mariani, Asunción Moreno, Jan Odijk, and Stelios Piperidis (Eds.). European Language Resources Association (ELRA), 1-7. http://www.lrec-conf.org/proceedings/ lrec2014/summaries/628.html

[6] Jeong-Won Cha, Jeen-Pyo Hong, and Chang-Uk Shin. 2017. Espresso : Korean Part of Speech Tagger. https://doi.org/10.5281/zenodo.884606

[7] Euisok Chung, Yi-Gyu Hwang, and Myung-Gil Jang. 2003. Korean named entity recognition using HMM and CoTraining model. In Proceedings of the Sixth International Workshop on Information Retrieval with Asian Languages, 2003, Sappro, Japan, Fuly 7, 2003. 161-167. https://dl.acm.org/citation.cfm?id=1118956

[8] CLARIN-D/SfS-Uni. Tübingen. 2012. WebLicht: Web-Based Linguistic Chaining Tool. Online. Date Accessed: 18 Jan 2019. URL https://weblicht.sfs.unituebingen.de/

[9] Hamish Cunningham, Diana Maynard, Kalina Bontcheva, Valentin Tablan, Niraj Aswani, Ian Roberts, Genevieve Gorrell, Adam Funk, Angus Roberts, Danica Damljanovic, Thomas Heitz, Mark A. Greenwood, Horacio Saggion, Johann Petrak, Yaoyong Li, and Wim Peters. 2011. Text Processing with GATE (Version 6). http://tinyurl.com/gatebook

[10] Victor de Boer, Johan Oomen, Oana Inel, Lora Aroyo, Elco van Staveren, Werner Helmich, and Dennis de Beurs. 2015. DIVE into the event-based browsing of linked historical media. Journal of Web Semantics 35 (2015), 152 - 158. https: //doi.org/10.1016/j.websem.2015.06.003 Semantic Web Challenge 2014.

[11] Richard Eckart de Castilho, Éva Mújdricza-Maydt, Seid Muhie Yimam, Silvana Hartmann, Iryna Gurevych, Anette Frank, and Chris Biemann. 2016. A Webbased Tool for the Integrated Annotation of Semantic and Syntactic Structures. In Proceedings of the Workshop on Language Technology Resources and Tools for Digital Humanities (LT4DH) at COLING 2016. 76-84. http://tubiblio.ulb.tu-darmstadt.de/ 97939/

[12] David Ferrucci and Adam Lally. 2004. UIMA: An Architectural Approach to Unstructured Information Processing in the Corporate Research Environment. Nat. Lang. Eng. 10, 3-4 (Sept. 2004), 327-348. https://doi.org/10.1017/ S1351324904003523
[13] Mark Hall, Eibe Frank, Geoffrey Holmes, Bernhard Pfahringer, Peter Reutemann, and Ian H. Witten. 2009. The WEKA data mining software: an update. SIGKDD Explorations 11, 1 (2009), 10-18.

[14] Sebastian Hellmann, Jens Lehmann, Sören Auer, and Martin Brümmer. 2013. Integrating NLP using Linked Data. In 12th International Semantic Web Conference. 21-25 October.

[15] Erhard Hinrichs, Marie Hinrichs, and Thomas Zastrow. 2010. WebLicht: WebBased LRT Services for German. In Proceedings of the ACL 2010 System Demonstrations. 25-29. http://www.aclweb.org/anthology/P10-4005

[16] Erhard Hinrichs and Steven Krauwer. 2014. The CLARIN Research Infrastructure: Resources and Tools for e-Humanities Scholars. Proceedings of the Ninth International Conference on Language Resources and Evaluation (LREC-2014) (May 2014), 1525-1531. http://dspace.library.uu.nl/handle/1874/307981

[17] Nancy Ide, James Pustejovsky, Christopher Cieri, Eric Nyberg, Denise Dipersio, Chunqi Shi, Keith Suderman, Marc Verhagen, Di Wang, and Jonathan Wright. 2016. The Language Application Grid. In Revised Selected Papers of the Second International Workshop on Worldwide Language Service Infrastructure - Volume 9442 (WLSI 2015). Springer-Verlag New York, Inc., New York, NY, USA, 51-70. https://doi.org/10.1007/978-3-319-31468-6 4

[18] Park Jungyeul. 2017. Berkeley parser model for Korean: Sejong treebank Berkeley parser model for Korean: Sejong treebank. https://doi.org/10.5281/zenodo.891267

[19] Clemens Neudecker and Georg Rehm. 2016. Digitale Kuratierungstechnologien für Bibliotheken. Zeitschrift für Bibliothekskultur 027.7 4, 2 (November 2016). http://0277.ch/ojs/index.php/cdrs_0277/article/view/158

[20] Joel Nothman, Nicky Ringland, Will Radford, Tara Murphy, and James R. Curran. 2013. Learning multilingual named entity recognition from Wikipedia. Artificial Intelligence 194 (2013), 151 - 175. https://doi.org/10.1016/j.artint.2012.03.006 Artificial Intelligence, Wikipedia and Semi-Structured Resources.

[21] Jungyeul Park. 2017. JHE Korean-English evaluation data. https://doi.org/10. 5281/zenodo.891295

[22] Jungyeul Park. 2017. MaltParser model for Korean: Sejong treebank. https: //doi.org/10.5281/zenodo.891273

[23] Antonis Lempesis Mark Greenwood Petr Knoth Richard Eckart de Castilho Stavros Sachtouris Byron Georgantopoulos Stefania Martziou Lucas Anastasiou Katerina Gkirtzou Natalia Manola Penny Labropoulou, Dimitris Galanis and Stelios Piperidis. 2018. OpenMinTeD: A Platform Facilitating Text Mining of Scholarly Content. In Proceedings of the Eleventh International Conference on Language Resources and Evaluation (LREC 2018) (7-12). European Language Resources Association (ELRA), Paris, France.

[24] Matic Perovsek, Janez Kranjc, Tomaz Erjavec, Bojan Cestnik, and Nada Lavrac. 2016. TextFlows: A visual programming platform for text mining and natural language processing. Sci. Comput. Program. 121 (2016), 128-152.

[25] Georg Rehm and Felix Sasaki. 2016. Digital Curation Technologies. In Special Issue of the Baltic fournal of Modern Computing (Vol. 4, No. 2) - Proceedings of the 19th Annual Conference of the European Association for Machine Translation (EAMT 2016). Riga, Latvia, 399.

[26] Georg Rehm, Julián Moreno Schneider, Peter Bourgonje, Ankit Srivastava, Rolf Fricke, Jan Thomsen, Jing He, Joachim Quantz, Armin Berger, Luca König, Sören Räuchle, Jens Gerth, and David Wabnitz. 2018. Different Types of Automated and Semi-Automated Semantic Storytelling: Curation Technologies for Different Sectors. In Language Technologies for the Challenges of the Digital Age: 27th International Conference, GSCL 2017, Berlin, Germany, September 13-14, 2017, Proceedings (Lecture Notes in Artificial Intelligence (LNAI)), Georg Rehm and Thierry Declerck (Eds.). Gesellschaft für Sprachtechnologie und Computerlinguistik e.V., Springer, Cham, Switzerland, 232-247. 13/14 September 2017.

[27] Georg Rehm, Julian Moreno Schneider, Peter Bourgonje, Ankit Srivastava, Jan Nehring, Armin Berger, Luca König, Sören Räuchle, and Jens Gerth. 2017. Event Detection and Semantic Storytelling: Generating a Travelogue from a large Collection of Personal Letters. In Proceedings of the Events and Stories in the News Workshop, Tommaso Caselli, Ben Miller, Marieke van Erp, Piek Vossen, Martha Palmer, Eduard Hovy, and Teruko Mitamura (Eds.). Association for Computational Linguistics, Vancouver, Canada, 42-51. Co-located with ACL 2017.

[28] Julian Moreno Schneider and Georg Rehm. 2018. Towards a Workflow Manager for Curation Technologies in the Legal Domain. In Proceedings of the LREC 2018 Workshop on Language Resources and Technologies for the Legal Knowledge Graph, Georg Rehm, Víctor Rodríguez-Doncel, and Julian Moreno Schneider (Eds.). Miyazaki, Japan, 30-35. 12 May 2018.

[29] Ray Smith and Google Inc. 2007. An overview of the Tesseract OCR Engine. In Proc. 9th IEEE Intl. Conf. on Document Analysis and Recognition (ICDAR. 629-633.

[30] Bo-Hyun Yun. 2007. HMM-Based Korean Named Entity Recognition for Information Extraction. In Knowledge Science, Engineering and Management, Zili Zhang and Jörg Siekmann (Eds.). Springer Berlin Heidelberg, Berlin, Heidelberg, 526-531.

[31] Amir Zeldes, Anke Lüdeling, Julia Ritz, and Christian Chiarcos. 2009. ANNIS: a search tool for multi-layer annotated corpora. In Proceedings of Corpus Linguistics. https://doi.org/10.18452/13437 